\title{
Providing Therapy and Educational Programs for Gifted Dyslexic Children Is a Challenge
}

\author{
Khalid Mohammed Almahrag \\ Special Education, King Saud University, Riyadh, KSA \\ Email:kalmahrej@ksu.edu.sa, almahrej@yahoo.com
}

How to cite this paper: Almahrag, K. M (2021). Providing Therapy and Educational Programs for Gifted Dyslexic Children Is a Challenge. Creative Education, 12, 2642-2656. https://doi.org/10.4236/ce.2021.1211197

Received: October 12, 2021

Accepted: November 22, 2021

Published: November 25, 2021

Copyright $\odot 2021$ by author(s) and Scientific Research Publishing Inc. This work is licensed under the Creative Commons Attribution International License (CC BY 4.0).

http://creativecommons.org/licenses/by/4.0/

\begin{abstract}
Children who are gifted and have learning disabilities possess a notable skill or aptitude and are capable of excellent performance, but also have a learning handicap that makes some aspects of academic attainment difficulty. We identify some of these children and provide them with the assistance they need. It occurs only rarely unless a school especially decides to identify and then serve these children. This paper presents a visualization of the essential points that must be dealt with to serve gifted children with dyslexia. The researcher has tried to describe children with dyslexia as gifted and has drawn heavily on giftedness and learning disabilities definitions. The idea of giftedness and learning disability is differently viewed and put forth by the researcher with the help of numerous research reviews. This research paper is helpful for teacher training institutes and educators to understand the characteristics and needs of gifted children with dyslexia and strategies to assist their learning.
\end{abstract}

\section{Keywords}

Gifted, Dyslexia, Providing Therapy

\section{Introduction}

The word dyslexia, which is of Greek origin, consists of two parts: days, meaning "difficulty," and Lexia, meaning "word" or "language." The International Dyslexia Association (IDA) (2002) defines dyslexia as a specific learning disability that is neurobiological in origin. There are difficulties with accurate and fluent word recognition, spelling, and decoding. Phonological difficulties usually cause language-related difficulties, often not predicted by other cognitive abilities, and can be addressed through effective classroom instruction. Secondary consequences may include reading comprehension problems and a limited reading 
experience, which can impede the growth of vocabulary and background knowledge.

Many people thought that there was a discrepancy when teachers initially started describing dyslexic youngsters who also appeared brilliant. Many individuals find it challenging to comprehend that a child can be both gifted and suffer from learning problems at the same time. Since Turman's (Silverman, 2002) time, the stereotype prevailed: gifted children score uniformly high on intelligence tests and do well in school. How could a child be considered gifted with serious enough learning problems to be characterized as having evidence of dyslexia? Therefore, children with dyslexia will usually not be identified and will not receive adequate treatment, despite their high abilities and learning disabilities. The lack of identification and underserving of children with dyslexia who share high abilities and learning difficulties is a significant issue. The concept of giftedness and learning difficulties coexisting in the same person has become widely acknowledged. On the topic, there have been many books and articles published in journals. Almost all educational conferences devoted to either learning disabilities or giftedness include a presentation on dual exceptionality; the question of dyslexic high-ability students has also received increasing attention over the last decade. Those gifted and talented disabled children need remedial learning activities. They also need opportunities to showcase their strengths and gifts in one or more fields where they have demonstrated their superior skills.

\section{Identification of gifted children with dyslexia}

The identification of gifted children with dyslexia is difficult due to the various definitions of giftedness. Dyslexic gifted children generally meet the eligibility criteria for giftedness and learning disabilities-the high achievement or I.Q. Scores of dyslexic children make them easy to identify them as gifted (Hannah \& Shore, 1995). Maybe they can build fantastic structures with plastic bricks or start a local campaign to save whales. Creative abilities, intellectual soundness, and passion for hobbies are clear indicators of giftedness (Winebrenner, 2002). While their verbal abilities impress teachers, their spelling and handwriting contradict this image. Frequently, they suffer from forgetfulness, carelessness, and inattention.

As opposed to students' specific progress, giftedness usually refers to high intellectual abilities. Generally, gifted children have exceptional learning abilities and problem-solving potential. It is a difficulty in cognitive processing that causes dyslexia, which is a problem in learning caused by a difficulty that affects only one or more cognitive processes rather than affecting the entire intellectual process. There is a persistent gap between their measured potential and their actual achievement in terms of academic duties (Hannah \& Shore, 1995). Children gifted with dyslexia receive exceptional educational support to overcome one or more handicapping conditions while also being prepared to excel in one or more fields in which they are talented (Whitmore, 1981). 
Aside from a depressed academic ability, these children also struggle with behavioral challenges and personality disorders. Children with such disorders typically have hearing or visual processing problems, attention deficit disorders, or difficulty following verbal instructions (Winebrenner, 2002). We still perceive gifted programs and special education services as mutually exclusive despite extensive research on talented children with dyslexia in the past decade. Most children fail to qualify for either program because identification protocols fail to recognize their unique characteristics to screen for learning disabilities among gifted dyslexic children. It is common to require documentation of underachievement or learning disabilities to qualify for either program. It is common to use the updated score patterns for the Wechsler Intelligence Scale for Children (WISC-R) when interpreting identification results for gifted children with dyslexia. Generally, the results of this research do not follow a consistent pattern. According to Schiff, Kaufman, and Kaufman (1981), there was a distinct Verbal-Performance (V-P) discrepancy between Verbal and Performance scores. Waldron and Saphire (1990) concluded that significant differences between Verbal and Performance scores might not indicate a learning disability in children. Schiff, Kaufman, and Kaufman concluded that the group of people with dyslexia with superior I.Q.s demonstrated above-average levels of spoken comprehension and expression skills and many original talents. However, they also indicated weaknesses in the cognitive area of sequencing, motor coordination activities, and emotional development. Waldron and Saphire claimed that these students depend on visual skills for word identification and analysis. In addition, they had poor performance in auditory areas, such as sound distinction and short-term memory.

According to Vaidya (1993), portfolio-type and creativity tests should be used with I.Q. and achievement tests to identify talented children with dyslexia. I.Q. Assessments should determine the learner's abilities and weaknesses, while achievement tests determine giftedness in a particular subject area. Portfolios should contain a child's ideas, drafts, criticisms, journal entries, final drafts, teacher comments, and parent suggestions to provide insight into the child's thinking process. She also advises the use of creativity tests that value divergent thinking.

The Torrance Tests of Creative Thinking evaluate fluency, flexibility, originality, and complexity (Winebrenner, 2002). In tests such as this one, a child's performance indicates his or her mental ability rather than the skills used in completing academic tasks. Achievement scores and I.Q. can be used based on Vaidya's (1993) ideas. However, they also suggested using the Scales for Rating the Behavioral Characteristics of Superior Students (SRBCSS) (Strop \& Goldman, 2002), for instance, creativity, learning, motivation, leadership, art, music, drama, and communications scales.

However, there is also debate about whether I.Q. Tests are the best or most appropriate evaluation of potential (Winebrenner, 2002). Recognizing a child's 
potential at a more problematic level can be helpful. The discussion pointed out that two children with very different I.Q.s. Scores, both struggling to learn to read, might not be significantly different in decoding skills. According to Lyon (Winebrenner, 2002), they are "qualitatively dissimilar from each other on tasks assessing a range of "intelligent" behaviors" that may be critical to their ability to learn and adapt.

Furthermore, a child's intelligence may influence their emotional and behavioral responses to constant failure, parent and teacher expectations, and, most importantly, remediation (Winebrenner, 2002). According to Olson (1985), people with dyslexia who rely heavily on heavy phonetic coding could read continuous texts more effectively by relying more on context and orthographic coding. For dyslexic children who are academically gifted, these arguments for recognizing their potential are essential.

There is some disagreement about using I.Q., achievement discrepancy to determine which children are dyslexic and academically gifted (Winebrenner, 2002). Identifying academically gifted students and learning disabilities requires considering the differences between performance and achievement. Even though a discrepancy between ability and achievement should not be the only characteristic of gifted children with dyslexia, part of the information should be carefully considered.

To identify gifted dyslexic children, one should look for evidence that they are gifted, talented, or capable of high achievement. In the case of dyslexia, teachers and sometimes even parents are not aware of the exceptional skills that the person possesses. Children with disabilities often hide their unique gifts and talents behind the mask of disability itself. In children, giftedness most frequently manifests itself in their spoken language and memory strategies. The ability to solve problems, be curious, and have the urge to learn is also characteristics of giftedness. Indicators of creativity are less specific and much more challenging to evaluate, but creativity is nonetheless an important indicator. It is crucial to determine the exactness of this indicator that cognitive capabilities are considered part of the creative process. It is best to look for applicants who produce innovative solutions or are highly motivated to engage in complex and lengthy creative activities, like writing a novel or creating a play (Reis \& McCoach, 2002). Dyslexic gifted children need an environment that nurtures their abilities while addressing their learning disabilities. To improve their inconsistent abilities, it is also essential to provide them with essential emotional support.

\section{Assessment and diagnosis of gifted children with dyslexia: \\ Cognitive tests}

In the entire world of cognitive tests, it is vital to know that different tests rely on different definitions of cognitive ability. Consequently, different tests may measure different skills and abilities. Administrators of cognitive tests should be aware of the methods and criteria used to select cognitive tests and view the scores as highly tentative assessments of learning ability (Wallace, Larsen, \& 
Elksnin, 1992).

In addition to the theory behind cognitive tests (such as distinguishing cognitive ability or developing cognitive ability tests), there is also the practical issue of their use. There is also debate over the impartiality and validity of the tests used to assess gifted children with dyslexia (Weinfeld, Robinson, Jeweler, \& Shevitz, 2002). Many cognitive measures have been misused, especially with African American children, Native Americans, or those who do not speak English, placed in schools for those with cognitive delays or learning disabilities based on their scores. Despite this, numerous court cases have involved the use of standardized cognitive tests to assess minority children. Evaluation professionals are becoming increasingly attentive to test bias, the importance of administering and interpreting standardized tests in a child's native language, and the necessity of combining information gathered from various sources with test scores. If we know for what purpose the tests do use, the question of what constitutes "cognitive ability" and the fairness of using measures of cognitive ability becomes less critical (Hannah \& Shore, 1995). When used to measure skills and abilities, cognitive tests are most helpful (and most appropriate). The ability to determine whether a gifted child with dyslexia possesses dyslexia and how that knowledge is applied to school programming

is another critical factor. Experience and verbal skills appear to be less critical for perception, processing speed, and spatial skills. Social judgment and common sense, numerical reasoning, concrete and abstract thinking, and the ability to recognize similarities and differences between objects or concepts, and vocabulary and language skills appear highly dependent on experience, training, and overall oral ability. Experience and verbal skills appear to be less critical for perception, processing speed, and spatial skills.

Cognitive tests can also provide valuable information about a child's ability to process information. Every person must take in, interpret, store, and retrieve information from memory accurately and efficiently to learn. Every individual can process some kind of information faster than others. While others struggle to draw stick figures, the artist can describe the world accurately. Using a combination of specific tones, the musician creates beautiful sounds. To create a mood, the writer combines words. Some of us are not good at any of these things. In school, gifted children with dyslexia need specific skills to function effectively. They must listen attentively so that other movements, sounds, or sights do not disturb them. It often requires children to hold multiple pieces of information in memory and to act upon them. To express themselves, they must find the right words and, eventually, commit these words to paper.

Cognitive tests can provide valuable information about a child's ability to process and retrieve information when appropriately interpreted. An individual cognitive test can provide a general measure of how well a child pays attention, processes information rapidly, distinguishes relevant information from less relevant details, orders events in chronological order, and remembers words. 
Kamphaus (1993) summarizes several research findings connected to the use of cognitive tests for assessment gifted children with dyslexia: School-aged Children have more stable cognitive scores than preschoolers, and gifted dyslexics have more stable scores than those without impairment; cognitive test scores can change from childhood to adulthood, likely, Social factors, economic factors, and environmental factors, values, Test scores are affected by family structure and genetics; factors such as low birth weight, malnutrition, anoxia (lack of oxygen), and fetal alcohol exposure hurt scores; and academic achievement is closely related to cognitive ability.

This last finding suggests that cognitive and achievement tests may not be so different and that cognitive tests may be considered specialized types of achievement tests (Kamphaus, 1993). It is steadfast with the suggestion that cognitive tests may best determine specific skills, abilities, and knowledge.

\section{Diagnostic and measurement bias}

To assess and diagnose gifted children with dyslexia must be identified their disorder at the beginning. There is no doubt that the demographics of American schools are changing. The number of children from ethnic, racial, or linguistic backgrounds that differ from the dominant culture steadily increases (National Center for Education Statistics, 1990). Many people have expressed concern regarding minority children overrepresenting special education programs and highly gifted children with dyslexia. Researchers have studied this in-depth. Multiple factors contribute to this, including bias against children from different cultural and linguistic backgrounds, especially the poor (Harry, 1992). The school's style and emphasis may also differ significantly from those found in the cultures of ethnically or linguistically diverse children. Culture and language influence learning and behavior, so the school system could misinterpret what children know, behave, or learn (Franklin, 1992). Education may lead educators to refer children for inappropriate assessments when they appear less capable than they are. Referrals may result in inappropriate assessment methods, leading to inappropriate conclusions and placements in particular education programs.

Additionally, many research and court decisions demonstrate that standardized tests (mainly cognitive and achievement tests) are culturally and linguistically biased against children from backgrounds that differ from the majority culture. On many tests, answering questions accurately too often depends upon having certain culturally based information or knowledge. Suppose children have not been exposed to that information through their culture or have not had the experiences to gain specific knowledge. In that case, they will not answer some questions or answer them in a way that is considered wrong within the majority culture. It can lead to inappropriate conclusions about children's ability to function within the school setting. Stampoltzis, Plakida, and Peristeri (2020) found that Phonological awareness and fast, automated name tests seem to be a reliable combination to identify students at risk of reading problems in the first grade. Because of this, when children come from a non-dominant culture or 
speak a language other than English, care must be taken when evaluating them. Because most cognitive, language and academic measures are developed based on the standards of the majority English-speaking culture, they may not be suitable for use with children from other cultures.

\section{The prereferral process}

According to Ortiz (1997), such children should first go through the pre-referral process. A prereferral process is usually required in many schools before an individualized evaluation takes place. The purpose of a prereferral is to determine whether the appropriate and adequate approach has been taken (Wallace, Larsen, \& Elksnin, 1992). It allows the school to arrange instruction or make other classroom modifications and address the noted problem. A prereferral process combines Direct Observation of students in the regular classroom, Observation of children in different settings, and Observation of how students interact verbally. Interviewing people who are familiar with the child is also essential. These individuals can provide plenty of information about his or her intents, adaptive behavior, Information processing methods, approaches to learning, and language skills. Interviewers need to realize how people's cultures and languages can affect how they interpret information. It will be helpful in planning, conducting, and interpreting a culturally sensory interview if we understand how people within that culture view disability, the educational system, and authority figures. It may be instrumental in gathering information from the home environment, which will help the assessment team understand the child within their own culture. Communication between parents and schools is essential. Parents should share their insights into their child's behaviors, attitudes, successes, and needs.

An in-depth language assessment must be conducted in both the child's native language and English before formal testing occurs. The examiner needs to understand that evaluating students in English when English is not their dominant language is highly misplaced. Translating texts from English is not an acceptable practice either. When an evaluator conducts a test or interview with a child, he or she must speak the child's language and be familiar with the child.

Examiners may have to use English-language instruments when tests or evaluation materials are not available in the student's native language. In controlling the test and interpreting results, examiners must be careful since this is prone to misinterpretation. It may be necessary to alter standard procedures for administering tests. It is possible to combine a paraphrase of instructions with explaining how to do a test task. Reading test items to a child instead of reading them allows them to review them more quickly than writing them down or consulting a dictionary (Wallace, Larsen, \& Elksnin, 1992). However, it is essential to realize that the standardization of tests must do broken if such changes do make, reducing their relevance and usefulness. A detailed report detailing the child's test performance should include all the changes made to the testing procedures. It is essential to interpret the test results carefully. Identifying gifted children with 
dyslexia at an early age is essential, yet current definitions of dyslexia make this problematic. Such tests as WISC III measure the difference between verbal I.Q. and performance I.Q., and sometimes the short-term memory through digital span. The Bangor test in EAL children (English is an Additional Language) is often under-identified. The appropriateness of I.Q./achievement discrepancy definitions does raise questions for the assessment of dyslexia in dyslexic children. For example, for EAL children with dyslexia, there may be additional complications, such as cultural background or difficulties with English, which may mask dyslexic problems. When looking for discrepancies, listening comprehension videos are recommended as an alternative to I.Q.

Nevertheless, this may also be unsuitable for children with dyslexia who are EALL. There are alternative definitions that emphasize reading accuracy and fluency or phonological processing skills. An excellent understanding of literacy development in EAL children will make presenting more accurate diagnoses and definitions easier.

Several studies on dyslexia in Semitic languages, such as Arabic and Hebrew, have found that children with dyslexia can comprehend phonological information poorly. Arabic speakers with and without dyslexia need to assess literacy and phonological processing skills.

The effects of self-esteem on dyslexic gifted children

Identity formation is influenced by multiple societal interactions (Hewitt, 2007). Teachers and parents influence children's self-evaluation (Riddick, 2001). Dyslexic children are more likely to have difficulties at school and socially, thus putting them at risk of developing distorted or damaged self-concepts (Burden, 2005; Coleman \& Hendry, 2002; Wortham, 2006).

Recent discussions in educational and psychological circles have focused on the concept of self-esteem. This discussion hinges on the question: "Does competence build self-esteem or does self-esteem build competence?" (Hannah \& Shore, 1995). Both sides of the discussion are accurate, so the discussion is irrelevant. Skill development and self-esteem are functionally related. A gifted child with dyslexia gains self-esteem and improves academic ability at the same rate as the other side of the equation. Moreover, as that competence increases, his self-esteem improves. Academic success is both a prerequisite and a consequence of positive self-esteem, as the careful and concerned caregiver must understand.

Casino, Llopis \& Llinares (2021) define self-esteem as accepting, connected, unique, powerful, and gifted. Self-esteem is a component of self-concept, which also includes self-image, ideal self, and self-esteem. As the two images of self-image and ideal self-do bring together, self-esteem does form. If the self-image is good and the ideal self is realistic, then the self-esteem is high. On the other hand, self-esteem is low if the self-image is lower or less than the ideal self. If self-esteem is high, we have self-confidence and can take risks and cope with the odd experience of failure. 
Self-esteem issues are significant for gifted children with dyslexia because self-assessment of this concept requires evaluating and comparing. Children with dyslexia have a hard time with these two skills. Because of this, these children have a difficult time measuring their self-esteem.

Casino, Llopis \& Llinares (2021) states that gifted dyslexic children will have high self-esteem: Feel capable of influencing another's views or behaviors positively, be able to communicate feelings and emotion in different situations, Approach new situations positively and assuredly, show a high level of frustration tolerance, accept the obligation, keep situation (positive and negative) incorrect perspective, communicate good feelings about themselves, Have a sense of control within themselves.

Dyslexic gifted children often suffer from low self-esteem, which hinders their development significantly. Dyslexic children struggle with self-esteem issues because they cannot read or spell as well as their peers. Feeling stupid, ugly, ignorant, or brainless is one example. The following behaviors commonly conceal low self-esteem: anger, self-criticism, crying, clowning behaviors, denial of problems, daydreaming and fantasy, and impassive behaviors. They endure the callous remarks of uniformed others, like, "If you are that smart, why can't you spell?" After hearing such a statement several hundred times (no, it is more like several thousand times), even the most competent individuals begin to doubt their intelligence.

Casino, Llopis \& Llinares (2021) note that gifted dyslexic children with low self-esteem will benefit from the program: Consistently communicate self-degrading statements, show learned helplessness, Not volunteer, Practice perfectionism, be very dependent, become easily defensive, show an excessive need for acceptance, have problems making decisions, show low frustration tolerance, Have little confidence in their judgment and be highly defenseless to peer pressure.

Franklin (1992) says there is a traditional focus on weakness and remediation of basic skills. An inciting educational environment that enables children to develop their talents fully needs to work on basic skills. It is crucial that enrichment activities encourage creativity and that weaknesses (like lousy handwriting or disorganization) do not get in the way of achievement and self-esteem. Gifted children who have learning difficulties do not realize and do not understand why they do not understand but know that they are not stupid. The development of healthy self-esteem is essential for gifted children with learning difficulties. When the situation is left unattended, it can spiral out of control. Children with dyslexia who fail more often may lash out at their frustrations and hurt their self-esteem. The more they act out, the more trouble and punishment they receive, further eroding their self-esteem. Having a sense of competence and love is crucial for a child to grow up. The development of self-esteem and relationships, which are essential for children with learning disabilities, may require more work on the part of parents (Franklin, 1992). Self-esteem and good relationships are areas worth developing as academic skills.

Home and school support for gifted children with dyslexia 
Providing support to a gifted child with dyslexia will boost his self-esteem, whether at school or home. However, Lithari (2019) indicated that the impact of school support on self-concept remains less than home support due to the inflated school requirements.

While gifted children with dyslexia may benefit from separate programs designed especially for them, their needs may be met by identifying their strengths and weaknesses and adapting existing school-based services to meet their needs. Gifted children with dyslexia need high-level special programming in their areas of strength. Individualized education programs can take advantage of this to meet the needs of gifted children with dyslexia who require accelerated and enriched instruction. When developing the child's unique educational program, the strengths and weaknesses of the child and the school's resources need to be considered (Strop \& Goldman, 2002). Specifications must consider the nature and severity of the child's disability and his or her level of giftedness. However, most experts emphasize that focusing on the child's strengths is more important than focusing on the weaknesses.

Many educationists who study gifted children with dyslexia have found that, ideally, they should read more often than their classmates. As a particular group, these children should receive instruction from a teacher sensitive to their academic, social, and psychological needs and from peers who are also afflicted with dual exceptionalities for at least a portion of the day. There is a lack of specific training for gifted children with dyslexia teachers, and there are few separate programs for gifted children with dyslexia. Hannah \& Shore (1995) report that some schools have created special classes for these children. It has been observed that some students stayed with their classmates all day; in other cases, students with giftedness and learning disabilities do bring to a resource room with peers who have similar abilities.

Several school systems identified gifted children with dyslexia in varying degrees and created a self-contained class for gifted children with severe dyslexia. The benefits of differentiated learning for children with special needs are numerous, regardless of their severity. Providing services in a mixture of gifted, special education, and general classrooms eliminates the moving from classroom to classroom required and may be better suited to meeting children's emotional needs. Academic achievement is usually enhanced with programs that influence motivation, raise self-esteem, and individualize instruction.

In Hawaii, ASSETS, a school for gifted dyslexic children, offers a full-time program for gifted children with dyslexia (Pierpont, 2006). School instruction is interdisciplinary and includes speeding up and enrichment to challenge the children, build essential skills, and address the children's emotional and social needs. It is also possible to provide academically gifted children with learning disabilities with a "part-time" resource room model that exposes such students to peers who have dual exceptionalities. According to literature describing these efforts, there have been several attempts to modify enrichment programs for 
disabled children. In one class where the children all identified as dyslexic gifted students, the Schoolwide Enrichment Model (Strop \& Goldman, 2002) utilizes. The program enables academically gifted children to undertake in-depth projects on topics of their choice, provided for students with dyslexia. The teacher was a gifted and special education specialist, and specific strategies compensate for their weaknesses and augment their disabilities. A gifted program model based on Betts' (1985) Autonomous Learner Model, which emphasizes enrichment in an atmosphere that encourages self-advocacy, has also been adapted to support gifted children with dyslexia.

Whether full-time or part-time, special classes for gifted children with dyslexia allow the teacher to develop a unique program for these children; challenging and presenting Weaknesses can be accommodated through structures and strategies. Children who live with other children who have seemingly opposing strengths and weaknesses gain a sense of support from each other.

When dyslexic gifted children return home from school, daily negative experiences can cause weariness and irritability. As a result, they may develop severe resentment if they do homework or receive extra tuition. Families may become tense when emotional and behavioral problems arise. The home can be a safe and happy place to learn, preventing failure, embarrassment, and bullying. Support from their parents will enable the student to learn without fear of looking silly in front of the class and teacher. This advantage is precious in cases of bullying or teasing at school. The general organizational difficulties of dyslexic children can also be tackled sympathetically at home. This advantage is precious in cases of bullying or teasing at school.

Parents need to choose a learning specialist care if they receive help outside of the public schools. Specialists should be able to explain things to parents in a way that they understand. As much as possible, the expert should be certified and have experience working with learners of the learner's particular age group and type of disability.

A parent is the best person to provide his or her child with the individualized instruction recommended by educational experts but nearly impossible to give at school. When parents and others know about the individual child, they can predict how they will experience the activities they provide. In some respects, parents who can get to know their young children as individuals can better help them learn than even the best teachers.

To facilitate effective home education, a home computer with a CD-ROM and Internet access will allow that access to be quick, easy, and valuable. With the use of word processing software and access to Email, even the most reluctant dyslexic may feel encouraged to communicate in writing, while gifted dyslexics can reveal their expertise.

\section{Conclusion}

There has been an attempt to describe gifted children with dyslexia by drawing 
heavily on giftedness and learning disabilities theories. There has not been sufficient consideration of the implications of these conditions overlapping. For example, the broad-based federal definitions of giftedness and other definitions recognize children's abilities in various areas. Therefore, a child with leadership or artistic talent will be labeled gifted and qualify for services even though he/she may not show aptitude in academic fields. If such a child also has dyslexia, he or she might be considered gifted and learning disabled. For most people, it is not difficult to accept or understand that children will have different abilities in art and math. Giftedness and learning disabilities are harder to accept when caused by related academic problems, such as excellent reading skills but poor spelling and writing skills.

Further, these two types of children (i.e., gifted and disabled) may reside in connected or unconnected areas. However, children with weaknesses in unconnected areas might be gifted and have a learning disability. Students who have both talents and disabilities in academic areas are likely to be underserved and need special services since their talents and disabilities overlap.

There should be an examination and expansion of descriptions of children who have academically gifted abilities and children who have dyslexia. These children exhibit both characteristics simultaneously in connected and unconnected areas. Most schools currently use the operational definitions to place children in gifted or special education programs, excluding many academically talented children with dyslexia who rarely meet the strict cut-offs of most identification procedures. The few children identified using existing definitions and guidelines usually mean acquiring services in one area, but not both.

The primary goal of special services for dyslexic children is to address their weaknesses. It may happen in the general classroom or a resource room for children with dyslexia. An expert who can offer remedial strategies to gifted dyslexics may be worthwhile for some time spent with them. Children with dyslexia who are also gifted will find an education resource room that is unlikely to be the best place to present intellectual stimulation. If the gifted child can spend part of the day in a learning disability resource room, this should be evaluated based on the nature, severity, and cause of the disability and the child's age. Children with more severe dyslexia are more likely to benefit from this placement. Educating teachers can make them more aware of their gifted children's needs, whose initial responsibility is to remedy deficiencies in their students.

Learning disabled and gifted children may be more common than anyone realizes. Because their educational needs are not acknowledged and addressed, these children suffer silently despite their high intellectual ability. Contrary to children with a learning disability accompanied by another handicap, dyslexic gifted children demonstrate a paradoxical picture in which exceptional strengths coexist with specific deficits (Hannah \& Shore, 1995). A gifted child with dyslexia, on the other hand, may be able to use the talents and abilities that they possess to compensate for their disability. Many students can overcome academic difficul- 
ties with support, understanding, and some educational intervention.

On the other hand, because they can draw on their strengths, the disability is masked for many children. At the same time, the "drag" on their academic performance averts them from consistently achieving at high levels. Consequently, these children are often not identified and end up being severely misunderstood and underserved by society.

There is a need to reevaluate current education regulations and practices to account for the differences between dyslexic and academically talented children. Our unique programming should not be defined based on fixed criteria or cut-off scores. It is necessary to develop a new model that does not use fixed criteria and cut-off scores. There is a need for a broader definition of giftedness and learning disability to accommodate children with exceptionalities. There should be flexible programming options to meet the individual needs of these children. Since human capacities are so diverse, it is safe to say that every youngster would benefit from a curriculum tailored to their own needs. However, this is especially important for gifted children with dyslexia, whose cognitive profiles are more variable than other children. Support for children's unique social and emotional needs who must deal with the significant inconsistencies in what they are and cannot do well is also significant. Teacher training is to help teachers understand the characteristics and needs of gifted children with dyslexia and strategies to assist their learning.

\section{Conflicts of Interest}

The author declares no conflicts of interest regarding the publication of this paper.

\section{References}

Betts, G. T. (1985). Autonomous Learner Model for the Gifted and Talented.

Burden, R. (2005). Dyslexia and Self-Concept: Seeking a Dyslexic Identity. Wiley-Blackwell.

Casino-García, A. M., Llopis-Bueno, M. J., \& Llinares-Insa, L. I. (2021). Emotional Intelligence Profiles and Self-Esteem/Self-Concept: An Analysis of Relationships in Gifted Students. International Journal of Environmental Research and Public Health, 18, Article ID: 1006. https://doi.org/10.3390/ijerph18031006

Coleman, J. C., \& Hendry, L. (2002). The Nature of Adolescence. Routledge.

Franklin, M. E. (1992). Culturally Sensitive Instructional Practices for African American Learners with Disabilities. Exceptional Children, 59, 115-122.

Hannah, C. L., \& Shore, B. M. (1995). Metacognition and High Intellectual Ability: Insights from the Study of Learning-Disabled Gifted Students. Gifted Child Quarterly, 39, 95-109.

Harry, B. (1992). Cultural Diversity, Families, and the Special Education System: Communication and Empowerment. Teachers College Press.

Hewitt, J. P. (2007). Self and Society: A Symbolic Interactionist Social Psychology (10th ed.). Pearson.

International Dyslexia Association (2002). Definition of Dyslexia. International Dyslexia 
Association https://dyslexiaida.org/definition-of-dyslexia/\#

Kamphaus, R. W. (1993). Clinical Assessment of Children's Intelligence: A Handbook for Professional Practice. Allyn \& Bacon.

Lithari, E. (2019). Fractured Academic Identities: Dyslexia, Secondary Education, Self-Esteem, and School Experiences. International Journal of Inclusive Education, 23, 280-296. https://doi.org/10.1080/13603116.2018.1433242

National Center for Education Statistics (1990). American Education at a Glance. National Center for Education Statistics, Office of Educational Research and Improvement, U.S. Department of Education.

Nielsen, M. E. (2002). Gifted Students with Learning Disabilities: Recommendations for Identification and Programming. Exceptionality, 10, 93-111. https://doi.org/10.1207/S15327035EX1002 4

Olson, R. K. (1985). Disabled Reading Processes and Cognitive Profiles. In D. Gray, \& J. Kavanagh (Eds.), Biobehavioral Measures of Dyslexia (pp. 215-244). York Press.

Ortiz, A. A. (1997). Learning Disabilities Are Occurring Concomitantly with Linguistic Differences. Journal of Learning Disabilities, 30, 321-332.

Pierpont, K. (2006). Our Greatest ASSETS. Teaching Pre K-8, 36, 42-45.

Reis, S. M., \& McCoach, D. B. (2002). Underachievement in Gifted and Talented Students with Special Needs. Exceptionality, 10, 113-125. https://doi.org/10.1207/S15327035EX1002 5

Riddick, B. (2001). Dyslexia and Inclusion: Time for a Social Model of Disability Perspective? International Studies in the Sociology of Education, 11, 223-236. https://doi.org/10.1080/09620210100200078

Schiff, M. M., Kaufman, A. S., \& Kaufman, N. L. (1981). Scatter Analysis of WISC-R Profiles for Learning Disabled Children with Superior Intelligence. Journal of Learning Disabilities, 14, 400-404.

Silverman, L. K. (2002). Upside-Down Brilliance: The Visual-Spatial Learner (pp. 302-305). DeLeon Publishing.

Stampoltzis, A., Plakida, E., \& Peristeri, E. (2020). Rapid Automatized Naming (RAN) and Its Relationship to Phonological Awareness and Reading: A Pilot Study in a Greek Sample of Students with Dyslexia. Open Journal of Modern Linguistics, 10, 174-194. https://doi.org/10.4236/ojml.2020.103011

Strop, J., \& Goldman, D. (2002). The Affective Side: Emotional Issues of Twice-Exceptional Students. Understanding Our Gifted, 14, 28-29.

Vaidya, S. R. (1993). Gifted Children with Learning Disabilities: Theoretical Implications and Instructional Challenge. Education, 113, 568-574.

Waldron, K. A., \& Saphire, D. G. (1990). An Analysis of WISC-R Factors for Gifted Students with Learning Disabilities. Journal of Learning Disabilities, 23, 491-498.

Wallace, G., Larsen, S. C., \& Elksnin, L. (1992). Educational Assessment of Learning Problems: Testing for Teaching. Allyn \& Bacon.

Weinfeld, R., Barnes-Robinson, L., Jeweler, S., \& Shevitz, B. (2002). Academic Programs for Gifted and Talented/Learning Disabled Students. Roeper Review, 24, 226-233. https://doi.org/10.1080/02783190209554185

Whitmore, J. R. (1981). Gifted Children with Handicapping Conditions: A New Frontier. Exceptional Children, 48, 106-114.

Winebrenner, S. (2002). Strategies for Teaching Twice-Exceptional Students. Understanding Our Gifted, 14, 3-6. 
Wortham, S. (2006). Learning Identity: The Joint Emergence of Social Identification and Academic Learning. Cambridge University Press.

https://doi.org/10.1017/CBO9780511810015 